\title{
NASA's Magnetic Gearing Research for Electrified Aircraft Propulsion
}

\author{
Dr. Justin J. Scheidler*, Vivake M. Asnani ${ }^{\dagger}$, and Thomas F. Tallerico \\ NASA Glenn Research Center, Cleveland, Ohio, 44135
}

\begin{abstract}
This paper presents an overview of the magnetic gearing research being conducted at NASA. First, the research is motivated through an in-depth discussion of the Agency-level aeronautics vision, the benefit of geared drivetrains, the barriers to applying mechanical gears in future aircraft concepts with electrified aircraft propulsion, and the characteristics of magnetic gears that allow them to overcome said barriers. Next, the principles of operation and current limitations of magnetic gears are discussed in the context of future electrified aircraft. Then, the authors' vision for the magnetic gearing research that will be needed to enable the next generation of electrified aircraft is presented. Lastly, the recent and ongoing magnetic gearing research at NASA is described, with a focus on the development and capabilities of a new rotating test rig and preliminary static measurements of a new in-house prototype. Results thus far are summarized.
\end{abstract}

\section{Introduction}

$\mathrm{T}$ o enable the next generation of fixed wing and vertical lift aircraft, NASA has set goals for aircraft efficiency, emissions, reliability, and noise [1]. In its recently updated aeronautics strategic plan [1], NASA noted the importance of parallel large and small aircraft development and also recognized the economic benefits of alternative propulsion. Specifically, electrified aircraft propulsion (EAP) was identified as a key enabler for low-cost, environmental-friendly, small aircraft for emerging markets, such as the short haul market.

To help meet its mid-term and long-term goals for fixed wing aircraft, NASA is investing in a broad portfolio of enabling research and development for EAP [2]. System-level studies have been performed on a number of vehicle concepts that use EAP. These studies have shown the feasibility of achieving system-level benefits to energy consumption, fuel burn, and/or emissions [3-9].

The EAP concepts that have been proposed span a broad array of payload, range, airframe configuration (e.g., distribution of the propulsion, amount of boundary layer ingestion), electrical power, and electrified propulsion system (turboelectric, parallel hybrid, all-electric, etc.). One commonality between all of the designs is the presence of electric machines (i.e., electric motors and, in some cases, generators). A design challenge of using electric motors to drive propellers is that, for a given power, the specific power and/or efficiency of an electric motor increases as its tip speed increases whereas the propulsive efficiency of a propeller increases as its tip speed decreases. Consequently, directly driving the propeller with an electric motor requires a compromise in speed between the two and an optimization of the propeller-motor system. An alternative is to connect the motor to the propeller through a speed reducing gearbox. This approach adds complexity and potentially reduces reliability but permits the motor and propeller to be optimized individually. A second challenge with the direct drive approach is that when noise is a concern, the propeller's tip speed must be reduced by slowing its rotation or reducing its diameter; in either case, the directly connected motor ends up occupying a larger fraction of the propeller's cross-sectional area, which reduces propulsive efficiency. With a geared motor, this penalty can be avoided by increasing the gear ratio to reduce the propeller's speed rather than decreasing the motor's speed, which would require a commensurate increase in its rated torque and diameter.

Mechanical power transmission in existing aircraft utilizes conventional mechanical gears that transmit torque through physical contact between mating gear teeth. This high-speed, high-pressure contact causes several problems, including energy loss, vibration-induced noise, tooth wear, and the possibility of tooth failure. In addition, a lubrication system is required to reduce friction and prevent gearbox failure due to a rapid buildup of heat in the teeth. Friction-induced heating becomes more severe as the torque and speed of a gear stage increases. Consequently, high power gearboxes (e.g., those in larger civilian rotorcraft) must be certified to survive for a certain amount of time under loss of lubrication [10-12];

\footnotetext{
*Research Engineer, Rotating and Drive Systems Branch, 21000 Brookpark Road, Cleveland, Ohio, 44135.

${ }^{\dagger}$ Research Engineer, Rotating and Drive Systems Branch, 21000 Brookpark Road, Cleveland, Ohio, 44135.

${ }^{\ddagger}$ Research Engineer, Rotating and Drive Systems Branch, 21000 Brookpark Road, Cleveland, Ohio, 44135.
} 
this commonly necessitates a backup lubrication system. Future subsonic transport and short haul aircraft that employ gears may be subjected to similar constraints.

In conventional transport aircraft, mechanical gears can be utilized in the aircraft's two to four large turbofans with a reasonable maintenance penalty. In many short haul concepts, the propulsion is distributed over several electric motors that could each benefit from a dedicated gearbox. However, mechanical gears are seldom implemented, because the imposed maintenance penalty would be costly. Gears that avoid the lubrication and maintenance limitations of mechanical gears can enable advances in the specific power and/or efficiency of electrified propulsion systems, particularly in short haul aircraft.

It was recognized over 100 years ago that magnets could be used to transfer torque through magnetic forces to prevent the physical contact between gear teeth [13]. Thus, magnetic gears eliminate the lubrication requirements, failure mechanisms, and reliability concerns of mechanical gears. Magnetic gears may also have a reduced vibration signature due to their lower mesh stiffness (lower force induced by motion transfer errors) and expected lower variation in mesh stiffness (smaller parametric excitation). Initially, magnetic gearing technology evolved slowly, because magnets were relatively weak and the designs were significantly heavier than mechanical gears for a given torque capacity. However, in the last 10 years, this technology has advanced substantially [14]. Commercial applications for the wind turbine and automotive industries have been developed. The state-of-the-art designs are lightweight enough to begin considering for aerospace applications [15].

The potential benefits of magnetic gearing for aerospace applications has prompted NASA to establish a 2-1/2 year project to develop a core competency in magnetic gearing. The project is divided into three phases. Phase one focuses on gaining a core understanding of the technology and increasing its specific torque (torque/mass). The second focuses on reducing the detrimental effect of high speed operation on the efficiency and specific torque of magnetic gears. The third phase explores the integration of magnetic gears into motors.

In the following section, the principles of operation and current limitations of magnetic gears are discussed in the context of future electrified aircraft. Then, the authors' vision for the magnetic gearing research that will be needed to enable the next generation of electrified aircraft is presented. Lastly, the recent and ongoing magnetic gearing research at NASA is described, with a focus on the development and capabilities of a new rotating test rig and preliminary static measurements of a new in-house prototype. Results thus far are summarized. This paper is similar to a recent one published by the authors [15], which focused on trends and lessons learned in prototype development for vertical lift applications. Here, an overview of that work is provided in the context of fixed wing applications along with a detailed description of the testing capabilities.

\section{Background}

To provide a framework for the discussions that follow, this section provides a brief summary of the history of magnetic gearing, their principles of operation, and their current limitations. For a broader and more thorough review, refer to [14, 16]. For a more in-depth discussion of the principles of operation, refer to [15].

\section{Principles of Operation}

The first magnetic gear was invented in 1901 [13]. Early designs (until the 1960s) resembled mechanical spur gears, but with each gear tooth replaced by a magnetic source. In most of the early designs, electromagnets were used as the magnetic source rather than permanent magnets, because the permanent magnets available at the time were significantly weaker than present day magnets, as demonstrated in Fig. 1. After the invention of higher strength magnets in 1966 and 1983, the use of permanent magnets became commonplace. Around the same time, new configurations were developed that achieved large improvements in torque capacity by simultaneously engaging multiple magnets. The concentric (coaxial) versions of these high-magnet-engagement designs evolved from simple analogs of mechanical planetary gear stages to out-of-the-box designs that harness the unique characteristics of magnetism and function like, but only partially resemble, mechanical planetary gears.

The current state of the art is a high-magnet-engagement design called the concentric magnetic gear (CMG), Fig. 2 . The CMG was invented in 1968 by Martin [18], but its potential was not realized until 2001 when Atallah and Howe developed the mathematics that enable its design for high torque density (torque/volume) [19]. The CMG has two permanent magnet members that contain different numbers of magnetic poles and behave like the ring and sun gears of a mechanical planetary. A flux modulator, located in-between the magnetic ring and sun gears, acts like the planet gears. It consists of alternating sections of ferromagnetic and non-magnetic material and uses a principle called flux modulation to allow all of the magnetic poles in the sun and ring to engage with one another in a way that produces a 


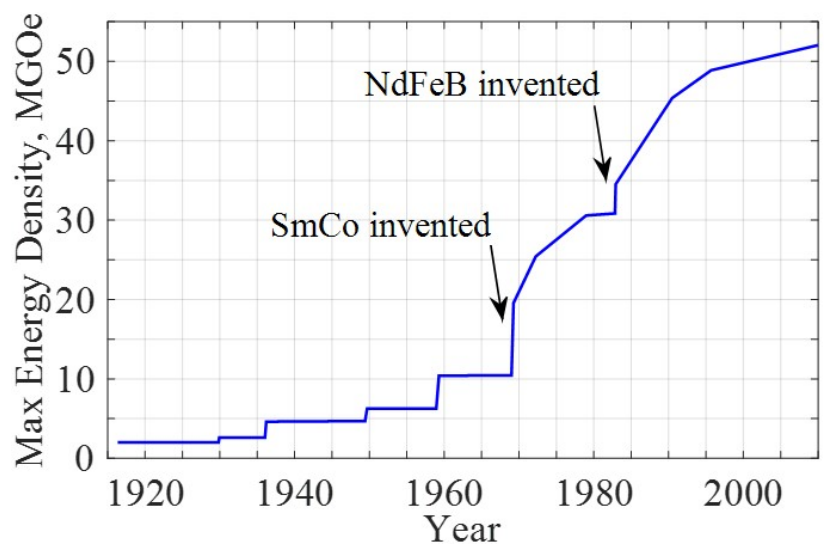

Fig. 1 Historical improvement in the maximum energy density of permanent magnets; data taken from [17].

gear ratio. This full engagement of all the magnetic poles enables the CMG to have higher torque density than other embodiments of magnetic gearing. Further improvements on the CMG's torque density were made by using Halbach arrays in both the sun and ring gears. A Halbach array combines multiple magnets in a specific orientation pattern to produce a focused and nearly sinusoidal magnetic flux field without the use of ferromagnetic materials [20]. Li et al. [16] numerically compared a CMG with Halbach arrays to other forms of concentric magnetic gearing and found it to have the highest torque density and lowest torque ripple. Currently, the CMG is the most developed form of magnetic gearing.
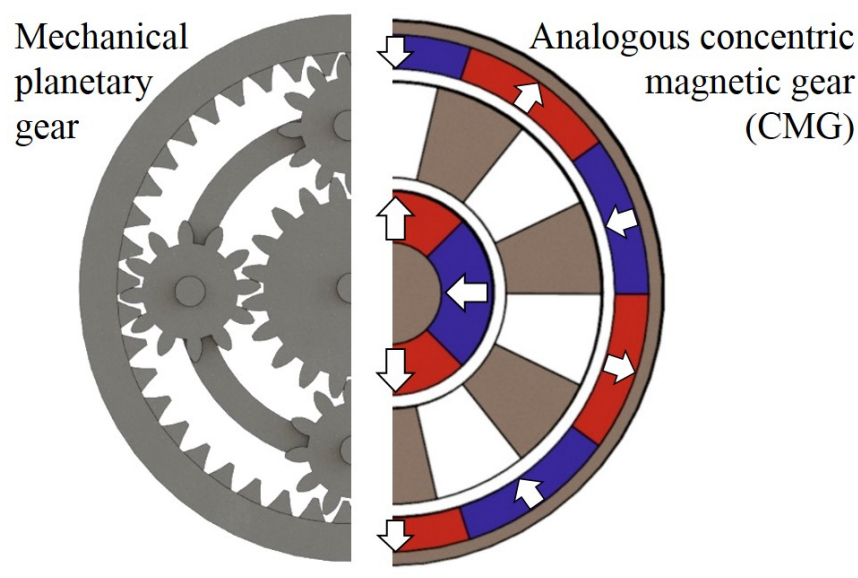

Fig. 2 Concentric magnetic gear (CMG) and its analogy to a mechanical planetary gear stage.

The 2D magnetic response of an example CMG is depicted in Fig. 3, where only the ring and flux modulator are included for explaining flux modulation. The closed loops indicate the paths taken by magnetic flux. The radial component of magnetic flux density is represented by color, where blue is inward and red is outward. Here, the ring gear is a Halbach array composed of 24 pole pairs, which each contain 6 magnets. The array produces a circumferentially-periodic flux pattern at its inner surface with a periodicity that is controlled by the number of magnetic pole pairs in the ring. The fundamental wavelength, or period of repetition, is $15^{\circ}$, or $360^{\circ} / 24$. this fundamental corresponds to a spatial harmonic order of 24 . The presence of the flux modulator causes some of the flux produced by the ring gear to project inside the flux modulator with a wavelength of $60^{\circ}$, or 4 times that of the ring's fundamental. The modulated flux waveform corresponds to a spatial harmonic order of 6 , as demonstrated in Fig. 3b. which depicts the radial flux density near the inner surface of the modulator. The spectrum includes strong 6th and 24 th order components, indicating only partial modulation of the ring's fundamental 24th order harmonic. The unintended higher harmonics that are present are caused by the ring itself and the modulator. The CMG produces a gear ratio based on how the modulated 6th order harmonic rotates as the ring gear is rotated. While the modulator is fixed, if the ring in Fig. 3a is 
rotated by one period $\left(15^{\circ}\right)$ so that pole piece $\mathrm{A}$ is centered on magnet 2 , rather than magnet 1 , the system reaches an identical position to that depicted. Thus, the modulated waveform must also rotate a full period $\left(60^{\circ}\right)$, or 4 times further than the ring. The CMG is completed by adding a sun gear that produces a 6th order harmonic that directly interacts with the modulated 6th order harmonic.

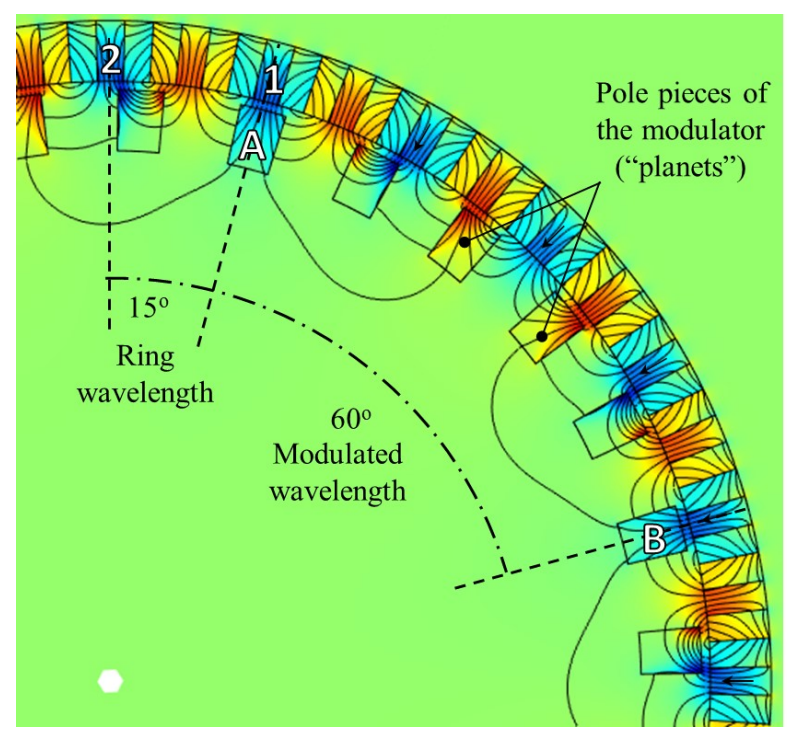

(a)

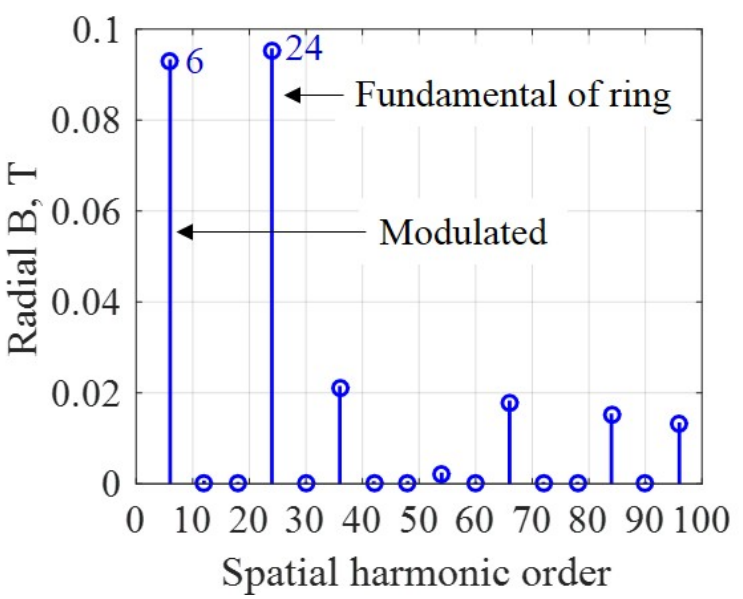

(b)

Fig. 3 Ring and modulator (analog of planets) of an example CMG with a 4:1 gear ratio: (a) 2D magnetic response from finite element simulation (closed black loops indicate the magnetic flux path and the colors indicate the radial component of the magnetic flux density) and (b) order domain representation of the spatiallydistributed radial magnetic flux density at the middle of the inner and outer air gaps.

The fundamental relationship that enables commensurate spatial harmonics to be produced is

$$
q=p_{l}+p_{h},
$$

where $q$ is the number of modulator pole pieces, and $p_{h}$ and $p_{l}$ are the number of pole pairs on the high and low speed rotors, respectively [14]. Like a planetary gear set, any of the members of the CMG may be fixed and the other two used as rotors. The gear ratio of the CMG is defined by the relative number of pole pairs in the same way that the relative number of gear teeth defines the gear ratio in mechanical gearing. If the modulator is the fixed member, the gear ratio is defined by the pole ratio according to

$$
G_{1}=\frac{p_{l}}{p_{h}},
$$

and the rotors spin in opposite directions. If the ring is fixed, the ratio is slightly higher,

$$
G_{2}=1+\frac{p_{l}}{p_{h}}=\frac{q}{p_{h}},
$$

and the rotors spin in the same direction.

\section{Research needs}

Despite having high potential for aeronautics applications, magnetic gearing is a relatively immature technology with a number of limitations that should be addressed. Most of these research needs arise from the different and often more demanding design criteria for the aeronautics applications of interest compared to the typical applications to date (wind energy, robotics, and automotive). Even for a given application, Gardner et al. demonstrated that there is a considerable variation in the optimal design of a CMG based on whether it is optimized to minimize volume, mass, or cost [21]. The two primary distinctions between most aeronautics applications and the contemporary applications are 
that the aeronautics applications demand minimal mass and higher speed (and usually higher efficiency). In most of the typical applications, the CMG is designed to maximize torque density.

Minimizing the mass of a prime mover-gearbox-propeller/fan system favors using a lower torque, higher speed prime mover, which requires a gearbox with a higher reduction ratio. The ratio of a mechanical planetary gear is fixed to the ratio of the ring and sun diameters; this is not a constraint for CMGs, as their ratio is determined by magnetic circuit design, which is only indirectly influenced by the diameters. More generally, it's not clear as to how the mass of magnetic gearing compares to mechanical gearing at various torque levels and gear ratios. The prototypes presented here show that the CMG can be mass-competitive at a relatively small-scale, but there is no theoretical basis or data set that enables this comparison to be extrapolated. This type of scaling information is important for identifying which applications are most suitable for the technology. A detailed study of the design tradeoffs associated with gear ratio would be insightful. Another existing limitation related to minimizing mass is the significant reduction (typically $20 \%$ to $40 \%$ [22]) in torque capacity that results from unintended axial leakage of magnetic flux. A better understanding is needed of how to design low-mass CMGs that also have low flux leakage.

Pushing the technology to higher speeds (in the 1,000s of rpm) while maintaining, or even improving, efficiency brings additional challenges. For CMGs to be competitive for aeronautics applications, it is likely that they will need to be at least as efficient over their entire operating envelope as the motors considered in system-level studies of concept aircraft (about 95\% to 99\% efficient). There are examples of prototypes that exhibit efficiency in this range but only for speeds in the 10s to 100 s of rpm. Research is needed on high speed efficiency modeling and characterization of magnetic gears, as well as the efficacy and tradeoffs of various loss reduction strategies (e.g., laminated magnets, ceramic magnets). Unless power loss can be kept very low, adequate heat dissipation will be critically important at high speed to ensure that magnets don't demagnetize. This issue should be considered fairly early in the design process. As speed increases, centrifugal and vibratory forces become significant. This is particularly important for the structure of the modulator and sun. Many CMGs utilize carbon fiber hoops to contain these components. At high speeds, this approach requires relatively thick hoops that limit performance by increasing the magnetic gaps. Further research on alternative containment designs can help to minimize the impact of speed on torque capacity. Also, more test data is needed to understand the high speed limits on efficiency for different materials systems.

\section{Research at NASA}

This section describes the recent and ongoing magnetic gearing research at NASA. Phase one of NASA's 2-1/2 year project on magnetic gearing was recently completed. The objective of phase one was to gain a core understanding of the technology and increase the specific torque. To accomplish this objective, two prototypes were built. The first (PT-1) was developed quickly with the goal of gaining practical magnetic gear design and manufacturing experience. The second (PT-2) was developed more methodically with the goal of maximizing specific torque. An overview of the development and a summary of the performance of these prototypes is provided here. For a detailed discussion and a complete list of lessons learned, refer to [15]. After the discussions about the prototypes, the development and capabilities of a new rotating test rig is discussed in detail.

\section{Prototype Development}

The prototypes were both loosely tailored to NASA's X-57 Maxwell aircraft's high-lift propulsors. Accordingly, their outer diameter was constrained to $152 \mathrm{~mm}$ and the structural components were designed to achieve an output speed of 4,500 rpm. A gear ratio of 4 was chosen to significantly reduce the torque, and thus mass, of the drive motor for each propulsor.

To support the rapid development of PT-1, it utilizes off-the-shelf magnets (N52 neodymium), electrical steel laminations in the modulator, and 3D printed structures (nylon-carbon fiber composite). The 3D printed material has sufficient strength and stiffness for this prototype, but it isn't suitable for a final design, because the printing tolerances are inadequate for minimizing physical gaps.

The design and components of PT-1 are depicted in Fig. 4 Two key design lessons were learned from this prototype. First, the use of off-the-shelf magnets lead to large magnetic gaps between adjacent magnets in the sun. Unsurprisingly, these gaps reduced the magnetic performance. Second, magnetic forces during assembly had a significant impact on the procedure. To avoid excessive deformation of some structures during assembly, the modulator, sun, and ring were first assembled individually and then the subassemblies were integrated. Shims were used between components during integration to prevent the magnets from contacting the pole pieces.

A multi-stage parametric study was used to refine the design of PT-2 and optimize its specific torque. Custom-shaped 

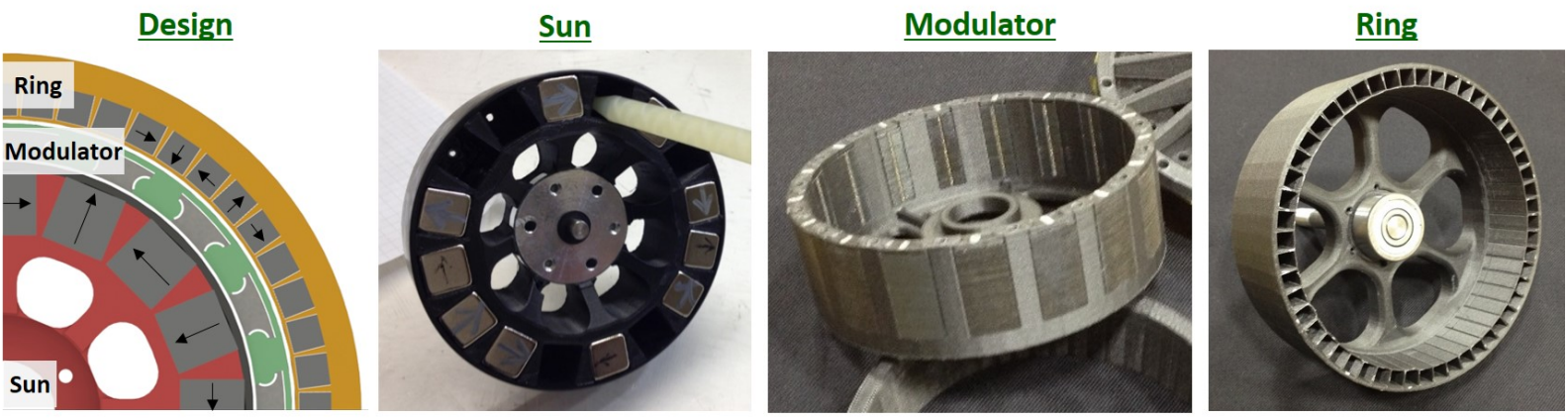

Fig. 4 Cross section of NASA's first prototype magnetic gear and images of each component at various stages of completion.

magnets were utilized to enhance the magnetic response. The design process revealed that the optimal thickness of the magnets and modulator are controlled by the thickness of the magnetic gaps. However, the minimum achievable magnetic gap is governed by the mechanical design. Consequently, mechanical design features that enable thinner magnetic gaps can improve specific torque. A cross section and image of PT-2 are shown in Fig. 5

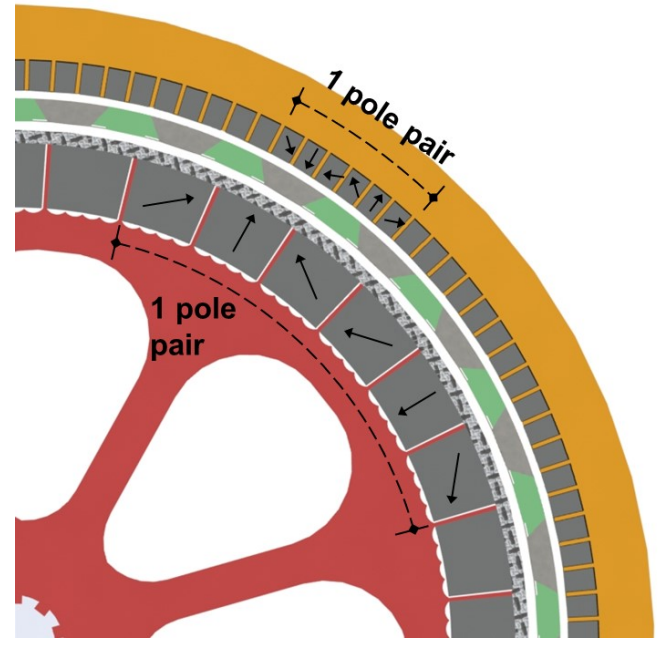

(a)

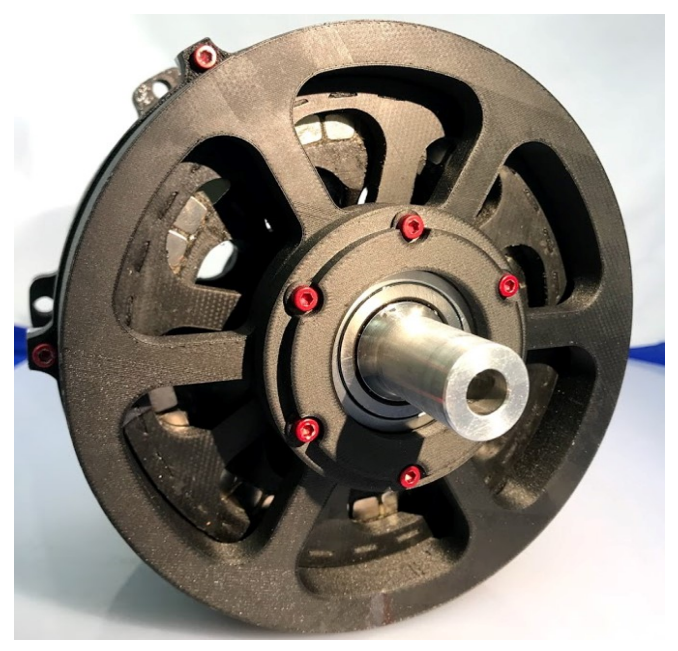

(b)

Fig. 5 NASA's second prototype magnetic gear: (a) cross section and (b) assembled prototype.

\section{Prototype Performance}

The mass and quasi-static performance of the prototypes is summarized in Table 1 . The measured torque capacity of PT-1 is 35\% lower than the 2D prediction. This level of degradation is reasonable considering the low length-to-diameter aspect ratio of PT-1 [22]. The assembly of PT-2 was just completed prior to publication of this paper. A preliminary static torque capacity measurement was obtained by fixing the sun (input shaft) and ring while rotating the modulator (output shaft) until it slipped from one magnetic pole to the next. The peak torque was recorded. Using this measurement, the preliminary specific torque of PT-2 is $44.7 \mathrm{Nm} / \mathrm{kg}$, which is over double that of PT-1 $(20 \mathrm{Nm} / \mathrm{kg})$. The primary design changes that led to this improvement are (a) the use of magnets shaped like annular wedges, which reduced the magnetic gaps between adjacent magnets, especially in the sun, (b) a unique containment structure for the modulator's magnetic lamination stacks that reduced the magnetic gap between the modulator and ring, (c) the specific flux of the Halbach magnet arrays was increased by increasing the number of magnets in each array from 4 to 6 and using radially-thinner, circumferentially-shorter, and higher pole count arrays, (d) thinner pole pieces that increased flux coupling and reduced mass. 
Table 1 Performance summary of PT-1 and PT-2.

\begin{tabular}{rcc}
\hline & PT-1 & PT-2 \\
\hline Torque, Nm & & \\
2D simulation & 53.0 & 178 \\
Measurement & 34.0 & 128 (preliminary) \\
Mass, kg & & \\
Active & $1.0(59 \%)$ & $1.7(59 \%)$ \\
Structural & $0.7(41 \%)$ & $1.2(41 \%)$ \\
Total & 1.7 & 2.868 \\
\hline Specific torque, Nm/kg & & \\
2D simulation & 31 & 61 \\
Measured & 20 & 44.7 (preliminary) \\
\hline
\end{tabular}

To understand how the performance of these prototypes compares to mechanical transmissions, Fig. 6 plots the specific torque of each prototype along with 61 aircraft transmissions (49 rotorcraft and 12 fixed wing aircraft), as well as a trend line that fits the aircraft data. The transmission data set was previously published in a different form for mass estimation [23]. The mass values used to compute specific torque include both the gearbox and lubrication system. As torque capacity reduces, aircraft transmissions become significantly less mass efficient. This strong trend motivates additional scaling studies for magnetic gears to develop a general comparison to mechanical transmissions and to identify the applications for which magnetic gearing is most suited. The conservative estimate of PT-2's specific torque is within $13 \%$ of the extrapolated trend line for aircraft transmissions, indicating that CMGs may be competitive with mechanical gearing in the near term for relatively low torque applications, such as the drive motors for small to moderate fixed wing concepts that have electrified propulsion systems. Before a conclusive comparison can be established, more magnetic gear prototypes need to be assessed and the TRL of NASA's prototypes needs to be elevated. In particular, PT-1 and PT-2 were not designed for continuous operation (heat transfer was neglected). Also, the suitability of a gearing technology for aeronautics applications also strongly depends on its efficiency. The magnetic gearing literature has established that highly efficient ( $>99 \%$ ) designs are possible, but more work is needed to determine if a sufficient combination of specific torque and high efficiency can be simultaneously achieved.

\section{Test Rig Development}

The purpose of the test rig is to study the components of an electrified drivetrain, including co-axial magnetic gears and electric motors. Accordingly, the test rig has been named the E-Drives Rig. The E-Drives Rig is a rotating system driven by a $30 \mathrm{~kW}(40 \mathrm{hp})$ electric motor and loaded by an eddy current dynamometer. Below is a CAD image of the rig with the key components labeled. The E-Drives Rig is similar to a typical in-line rotating test rig, but it has three specialized features

1) Adaptability - The E-Drives Rig has a high degree of adaptability. The components are mounted onto a table that has a bolt pattern with $25.4 \mathrm{~mm}(1 \mathrm{in})$ hole spacing. This allows for the testing of a wide variety of test articles, including drivetrain components for which the input and output shafts have a parallel offset or (with minor modifications) a slight angular offset.

2) Very high precision - The primary measurement of interest is efficiency. There are multiple ways of quantifying the efficiency of a gearbox using a combination of mechanical, electrical, and/or thermal measurements. The E-Drives Rig uses the direct approach: measuring torque and speed at the input and output and calculating power and efficiency. This approach requires few components, avoids complicated or highly uncertain calibration procedures, eliminates the influence of many secondary components (e.g., motor losses, heat leak), and is minimally affected by changes in the ambient environment. However, for high efficiency systems (magnetic gears can reach 99\%), it is difficult to measure torque and speed with sufficient precision to achieve a low uncertainty in the calculated efficiency due to error propagation. This issue is overcome by using the most precise torque transducers available $(< \pm 0.02 \%$ total uncertainty in torque). The torque transducer is further discussed below. 


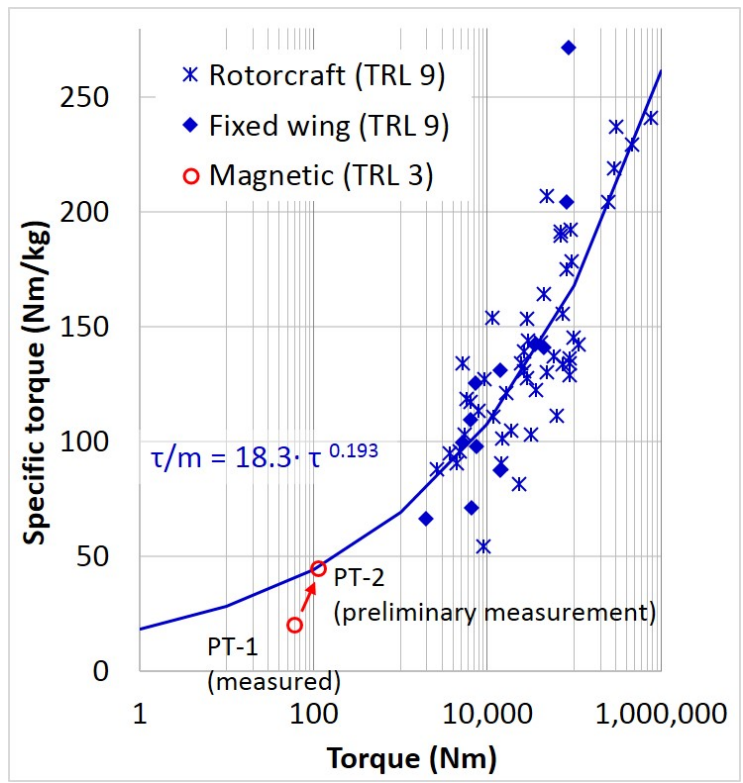

Fig. 6 Specific torque of TRL 9 aircraft transmissions and TRL 3 CMG prototypes.

3) Dynamic measurement - The E-Drives Rig is designed for studying vibration and transient responses. This is achieved through structural design and hardware selection. The vibration modes of the rig's rotating system that are within the measurement bandwidth will alter and obscure a measurement of the test article's dynamic response. To push the vibration modes of the rig's rotating system to higher frequencies, each component was designed or selected to be as lightweight and stiff (both torsionally and transversely) as possible. Hollow shafts of minimal length are used along with torsionally stiff disc couplings. The selected disc couplings also have zero backlash to prevent nonlinear vibration. Also, the components are installed on a vibration isolation table, which should eliminate the influence of floor vibrations and help to reduce the vibration transmitted from one component to another through the support structure. The measurement hardware for mechanical quantities was selected to permit a measurement bandwidth of $6 \mathrm{kHz}$, which will allow for the measurement of torque ripple up to a few harmonics of the expected fundamental electrical frequency of the CMG's high speed rotor.

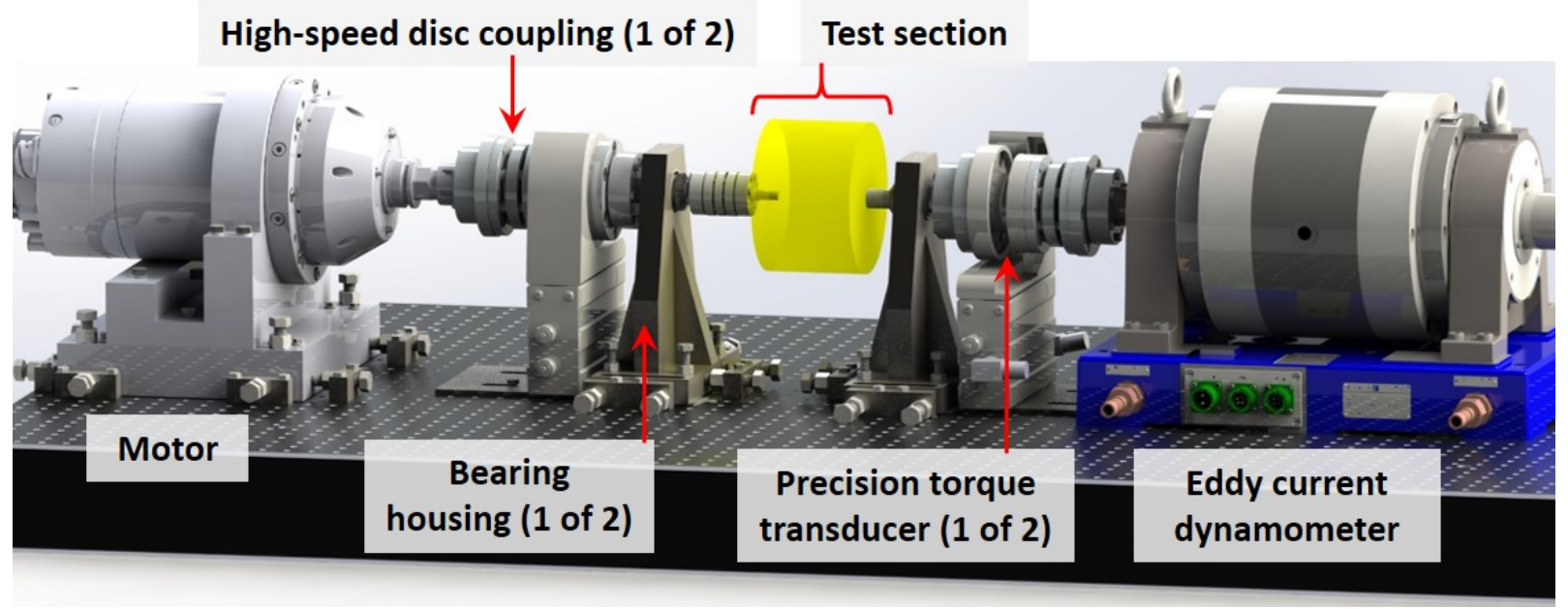

Fig. 7 CAD model of the high-speed electrified drive system test rig being installed at NASA Glenn Research Center.

The key specifications of the E-Drives Rig are summarized in Table2. The tabulated uncertainties for power and 
efficiency were calculated using error propagation equations that consider the uncertainty in the measured quantities from which the power and efficiency are calculated as well as the influence of the calculation itself [24]. These uncertainty calculations assume a speed measurement uncertainty of $\pm 0.01 \%$, which is a reasonable assumption for a high precision encoder measurement. A future publication dedicated to the development of this rig will include updated calculations with actual speed measurement uncertainties.

Table 2 Key specifications of NASA's new electrified drive system test rig (E-Drives Rig); the uncertainty calculations assume a speed measurement uncertainty of $0.01 \%$.

\begin{tabular}{|c|c|c|}
\hline Test article max size & $\begin{array}{r}\text { OD, cm (in) } \\
\text { Axial length, cm (in) }\end{array}$ & $\begin{array}{l}35.6(14) \\
30.5(12)\end{array}$ \\
\hline \multirow{3}{*}{ Input } & Max continuous torque, $\mathrm{Nm}(\mathrm{ft}-\mathrm{lb})$ & $16(11.8)$ \\
\hline & Max speed, rpm & 22,000 \\
\hline & Max continuous power, $\mathrm{kW}(\mathrm{hp})$ & $30(40.2)$ \\
\hline \multirow{3}{*}{ Output } & Max continuous torque, $\mathrm{Nm}(\mathrm{ft}-\mathrm{lb})$ & $100(73.7)$ \\
\hline & Max speed, rpm & 15,000 (with minor balancing) \\
\hline & Max continuous power, $\mathrm{kW}(\mathrm{hp})$ & $30(40.2)$ \\
\hline \multirow{6}{*}{$\begin{array}{l}\text { Measurement accuracy at } \\
\text { nominal state (not including } \\
\text { parasitic losses or the effects } \\
\text { of misalignment }\end{array}$} & Torque, $\%$ & \pm 0.02 \\
\hline & Average power, \% & \pm 0.08 \\
\hline & Instantaneous power, $\%$ & \pm 0.05 to \pm 0.13 \\
\hline & Average efficiency, \% & \pm 0.11 \\
\hline & Instantaneous efficiency, $\%$ & \pm 0.13 \\
\hline & Measurement bandwidth, $\mathrm{kHz}$ & 6 \\
\hline
\end{tabular}

Operating the E-Drives Rig involves controlling the motor's speed and the dynamometer's torque or power. Motor control is accomplished with an industrial variable speed AC drive and its associated programmable logic controller. The dynamometer is excited by the current output from a linear amplifier. The amplifier is commanded by a real-time control system to control the dynamometer in a closed loop; the loop is closed by feeding back the torque measured by adjacent precision torque transducer rather than the dynamometer's built-in torque sensor, which has only modest precision.

Multiple options exist for loading a drivetrain. An eddy current dynamometer was selected, because it provides adequate torque over a range of speed that can emulate moderate to high speed propellers (about 1,000 to 4,000 rpm), has a high maximum rotational speed, and is relatively compact. The downside of this option is that its torque capacity decays quickly as speed is reduced below $1,000 \mathrm{rpm}$, such that it has limited usefulness below about $650 \mathrm{rpm}$. Some aircraft concepts with electrified propulsion systems have propellers that spin slowly (about 400 to 1,000 rpm) to limit community noise. For emulating these loads, it may be necessary to replace the eddy current dynamometer with a magnetic hysteresis dynamometer or a generator.

Several digital signals (torque, speed, and average power) and analog signals (temperature and vibration) will be measured under transient and steady-state conditions for motor speeds up to 22,000 rpm. Except for the temperature signals, the analog measurements are recorded at $20 \mathrm{kHz}$. Each signal is passed through an anti-aliasing filter prior to aquisition. For testing magnetic gears, the core measurements are efficiency and torque (capacity, ripple, and response to overload) as functions of the rotational speed. As explained above, very high precision sensors were selected for these measurements. To achieve the high precision, the torque transducers are telemetry-based (non-contact) and have frequency outputs rather than typical voltage outputs. Instantaneous power and efficiency can be calculated from the torque and speed signals up to the transducers' bandwidth of $6 \mathrm{kHz}$. The transducers also output a power signal at a low sampling rate, which can be used for calculating average efficiency with higher precision than the instantaneous efficiency. Accelerometers are used to measure the translational vibration of the test rig and test article and can be used to observe the vibration signatures of the prototypes. A triaxial ICP accelerometer with $7 \mathrm{kHz}$ bandwidth is stud mounted to the top of each bearing housing to monitor the health of the bearings and to detect excessive vibration. Two or more 
unidirectional ICP accelerometers with $10 \mathrm{kHz}$ bandwidth will be stud mounted to the outer surface of the test article's housing. Since the bearing housings are located between the torque transducers and the test article, the calculated efficiencies will include bearing losses. Despite reducing these losses through the use of super precision hybrid ceramic bearings with low contact angle, the bearing losses are expected to be appreciable (relative to the low uncertainty in efficiency) within a portion of the operating space. To mitigate this concern, the bearing losses will be measured for a variety of temperature and speed conditions without a test article installed. This tare loss will be subtracted from calculated efficiencies to determine the efficiency of only the test article. To improve the determination of the tare loss, the temperature of each set of bearings will be measured with a spring-loaded resistance temperature detector that maintains contact with a bearing's outer race throughout operation of the rig. The electrical measurements include the aforementioned control signals for the motor and dynamometer and monitoring signals for the dynamometer's amplifier.

To correctly compensate for misalignment in the driveline, one dual flex coupling is required between adjacent components that are rigidly mounted to the support table. Several types of flexible couplings are available. Here, disc couplings were selected, because they provide the best combination of characteristics: excellent torque-to-outer-diameter ratio, high torsional stiffness, excellent speed capability, zero backlash, and low reactionary loads due to misalignment. The only notable downside is that disc couplings have low misalignment capability. This is important, because it constrains the allowable runout of the test article's shafts, making their design and fabrication more challenging.

\section{Conclusions and Future Work}

Magnetic gearing is an alternative to mechanical gearing, where torque is transferred through magnetic force as opposed to contact force. The technology has the potential to be used in aircraft applications, without the lubrication, noise, and maintenance issues associated with mechanical gearing. NASA has initiated a 2-1/2 year project to establish a core competency and advance this technology.

This paper summarized the progress made in the first six months of the project, where the torque-to-mass ratio (specific torque) of concentric magnetic gear (CMG) technology was examined. This was done through the development of two small-scale (<152 mm diameter) prototypes. Prototype 1 (PT-1) was developed rapidly to gain an understanding of design and fabrication issues. Its predicted specific torque (2D finite element simulation) was $31 \mathrm{Nm} / \mathrm{kg}$ while its measured value was $20 \mathrm{Nm} / \mathrm{kg}$. Prototype 2 (PT-2) was developed using a multi-stage parametric study to achieve a design with near optimal specific torque. The predicted specific torque and preliminary measurement of PT-2 are $61 \mathrm{Nm} / \mathrm{kg}$ and $44.7 \mathrm{Nm} / \mathrm{kg}$, respectively. To begin understanding how suitable CMGs are for aeronautics applications, the specific torque of PT-1 and PT-2 were compared to several rotorcraft and fixed wing mechanical transmissions. The preliminary specific torque of PT- 2 was found to be within $4.2 \%$ of the extrapolated trend line for the aircraft transmissions, indicating that CMG technology is viable for aeronautics, at least for low torque applications. Several design tradeoffs were established, as detailed elsewhere [15]. It was found that magnetic gaps dictate the achievable specific torque and the optimal geometry for the magnetic components. Moreover, the magnetic gaps are controlled by the shape of magnets that are used and the structure that holds and contains the magnetic components. Hence, specific torque can be further improved if (a) custom-shaped magnets are utilized and (b) if novel structural designs are implemented to minimize the containment structure located in the magnetic gaps or to improve the integration of magnetic and structural components.

The development and capabilities of a new rotating test rig called the E-Drives Rig were discussed in detail. The purpose of the test rig is to study the components of an electrified drivetrain, including co-axial magnetic gears and electric motors, in a rotating system. The E-Drives Rig is a $30 \mathrm{~kW}$ (40 hp) rotating driveline driven by an electric motor and loaded by an eddy current dynamometer. Its unique or less common features include (1) the ability to test a wide variety of test articles via a highly adaptable placement of the motor, dynamometer, and bearing housings, (2) the ability to directly measure the mechanical efficiency of the test article with very high precision (around $\pm 0.1 \%$ uncertainty), and (3) the ability to measure dynamic responses and mechanical vibration of the test article up to high frequencies with minimal influence from the test rig. The key specifications of the E-Drives Rig were discussed along with the rig's control system, the collection of measurements that will be obtained, and important decisions on design and hardware selection.

NASA's future research and development of magnetic gears will continue with phases two and three of the aforementioned 2-1/2 year project. The second phase will focus on reducing the detrimental effect of high speed operation on the efficiency and specific torque of magnetic gears. Specifically, the research will focus on three aspects:

data, design, and materials. The current lack of data at high speed operation will be addressed by using the E-Drives Rig to measure the speed dependence of torque, efficiency, vibration, and temperature. Design studies will be conducted 
to reduce the source of the key high speed loss mechanism (eddy currents) and to reduce magnetic gaps with novel design and manufacturing solutions for magnet and modulator containment. An exploration of alternative or laminated magnetic materials and electrically-insulating, thermally-conductive structural materials will be carried out. The third phase of the project will explore the integration of magnetic gears into motors. This will include application to short haul and urban air mobility aircraft.

\section{Acknowledgments}

This work was supported by NASA's Internal Research and Development (IRAD) and Revolutionary Vertical Lift Technology (RVLT) Projects. The authors would like to acknowledge Dr. Tim Krantz from NASA Glenn Research Center for providing the database of performance specifications of TRL 9 rotorcraft and fixed wing gearboxes.

\section{References}

[1] NASA Aeronautics Research Mission Directorate, “Strategic Implementation Plan 2017 Update,”, 2017.

[2] Jansen, R., Bowman, C., Jankovsky, A., Dyson, R., and Felder, J., "Overview of NASA Electrified Aircraft Propulsion (EAP) Research for Large Subsonic Transports," 53rd AIAA/SAE/ASEE Joint Propulsion Conference, AIAA Propulsion and Energy Forum, Atlanta, GA, AIAA 2017-4701, 2017. doi:10.2514/6.2017-4701.

[3] Bradley, M. K., and Droney, C. K., "Subsonic Ultra Green Aircraft Research: Phase I Final Report," NASA Contractor Report, NASA/CR-2011-216847, 2011. URL https://ntrs.nasa.gov/search.jsp? $\mathrm{R}=20110011321$ \&hterms=NASA\%2fCR-2011-216847\&qs=N\%3DQ\%26Ntk\%3DAl1\%26Ntt\%3DNASA\%252FCR-2011216847\%26Ntx\%3Dmode\%2520matchallpartial

[4] Felder, J. L., Brown, G. V., DaeKim, H., and Chu, J., “Turboelectric Distributed Propulsion in a Hybrid Wing Body Aircraft," 20th International Society for Airbreathing Engines, ISABE-2011-1340, Gothenburg, Sweden, 2011. URL https://ntrs.nasa.gov/search.jsp?R=20120000856.

[5] Bradley, M. K., and Droney, C. K., "Subsonic Ultra Green Aircraft Research: Phase 2. Volume 2; Hybrid Electric Design Exploration," NASA Contractor Report, NASA/CR-2015-218704/VOL2, 2015. URL https://ntrs.nasa.gov/search.jsp?R=20150017039\&hterms=NASA\%2fCR-2015-218704\%2fV0L2\&qs= N\%3D0\%26Ntk\%3DAll\%26Ntt\%3DNASA\%252FCR-2015-218704\%252FVOL2\%26Ntx\%3Dmode\%2520matchallpartial.

[6] Lents, C., Hardin, L., Rheaume, J., and Kohlman, L., "Parallel Hybrid Gas-Electric Geared Turbofan Engine Conceptual Design and Benefits Analysis," 52nd AIAA/SAE/ASEE Joint Propulsion Conference, AIAA Propulsion and Energy Forum, Salt Lake City, UT, AIAA 2016-4610, 2016. doi:10.2514/6.2016-4610.

[7] Schiltgen, B. T., Freeman, J. L., and Hall, D. W., "Aeropropulsive Interaction and Thermal System Integration within the ECO-150: A Turboelectric Distributed Propulsion Airliner with Conventional Electric Machines," 16th AIAA Aviation Technology, Integration, and Operations Conference, AIAA AVIATION Forum, Washington, D.C., AIAA 2016-4064, 2016. doi:10.2514/6.2016-4064.

[8] Welstead, J., and Felder, J. L., "Conceptual design of a single-aisle turboelectric commercial transport with fuselage boundary layer ingestion," 54th AIAA Aerospace Sciences Meeting, AIAA SciTech Forum, San Diego, CA, AIAA 2016-1027, 2016. doi:10.2514/6.2016-1027.

[9] Perullo, C., Trawick, D., Armstrong, M., Tai, J. C., and Mavris, D. N., "Cycle Selection and Sizing of a Single-Aisle Transport with the Electrically Variable Engine(TM) (EVE) for Fleet Level Fuel Optimization," 55th AIAA Aerospace Sciences Meeting, AIAA SciTech Forum, Grapevine, TX, AIAA 2017-1923, 2017. doi:10.2514/6.2017-1923.

[10] European Aviation Safety Agency, “Rotorcraft gearbox loss of lubrication,” NPA 2017-07, 2017.

[11] Federal Aviation Administration, “Airworthiness Standards: Transport Category Rotorcraft,” FAR 29.927(c), 2017.

[12] Federal Aviation Administration, “Airworthiness Standards: Normal Category Rotorcraft,” FAR 27 Appendix C, 2017.

[13] Armstrong, C., "Power transmitting device,” US Patent \# 687,292, 1901.

[14] Tlali, P., Wang, R., and Gerber, S., "Magnetic gear technologies: A review," Proc. of 2014 Intl. Conf. on Elec. Machines (ICEM), IEEE, 2014, pp. 544-550. 
[15] Asnani, V., Scheidler, J., and Tallerico, T., "Magnetic gearing research at NASA," Proc. of AHS Intl. 74th Annual Forum, Phoenix, AZ, May 14-17, 2018.

[16] Li, X., Chau, K., Cheng, M., and Hua, W., "Comparison of magnetically-geared permanent magnet machines," Progress in Electromagnetics Research, Vol. 133, 2013, pp. 177-198.

[17] Constantinides, S., "The demand for rare Earth materials in permanent magnets," 51st Annual Conf. of Metallurgists, Niagara Falls, Canada, 2012.

[18] Martin Jr., T., “Magnetic transmission,” US Patent \#3,378,710, 1968.

[19] Atallah, K., and Howe, D., “A novel high-performance magnetic gear,” IEEE Transactions on Magnetics, Vol. 37, No. 4, 2001, pp. 2844-2846. doi:10.1109/20.951324.

[20] Halbach, K., "Design of permanent multipole magnets with oriented rare earth cobalt material," Nuclear Instruments and Methods, Vol. 169, No. 1, 1980, pp. 1-10. doi:10.1016/0029-554X(80)90094-4.

[21] Gardner, M., Jack, B., Johnson, M., and Toliyat, H., "Comparison of coaxial radial flux magnetic gears independently optimized for volume, cost, and mass," 2017 IEEE International Electric Machines and Drives Conference (IEMDC), IEEE, 2017, pp. 1-8. doi:10.1109/IEMDC.2017.8002248.

[22] Gerber, S., and Wang, R., "Analysis of the end-effects in magnetic gears and magnetically geared machines," 2014 International Conference on Electrical Machines (ICEM), IEEE, 2014, pp. 396-402. doi:10.1109/ICELMACH.2014.6960211.

[23] Brown, G., Kascak, A., Ebihara, B., Johnson, D., Choi, B., Siebert, M., and Buccieri, C., "NASA Glenn Research Center Program in high power density motors for aeropropulsion,” Tech. rep., NASA TM 213800, 2005.

[24] Figliola, R., and Beasley, D., Theory and design for mechanical measurements, $4^{\text {th }}$ ed., John Wiley \& Sons, Inc., Hoboken, NJ, 2015. 